\title{
Cloud-Based Battery Condition Monitoring and Fault Diagnosis Platform for Large-Scale Lithium-Ion Battery Energy Storage Systems
}

\author{
Taesic Kim * (D), Darshan Makwana, Amit Adhikaree, Jitendra Shamjibhai Vagdoda and Young Lee \\ Department of Electrical Engineering and Computer Science, Texas A\&M University-Kingsville, MSC 192, \\ 700 University Blvd, Kingsville, TX 78363, USA; darshan.makvana@students.tamuk.edu (D.M.); \\ amit.adhikaree@students.tamuk.edu (A.A.); jitendra.vagdoda@students.tamuk.edu (J.S.V.); \\ young.lee@tamuk.edu (Y.L.) \\ * Correspondence: taesic.kim@tamuk.edu; Tel.: +1-361-593-4851
}

Received: 15 December 2017; Accepted: 2 January 2018; Published: 4 January 2018

\begin{abstract}
Performance of the current battery management systems is limited by the on-board embedded systems as the number of battery cells increases in the large-scale lithium-ion (Li-ion) battery energy storage systems (BESSs). Moreover, an expensive supervisory control and data acquisition system is still required for maintenance of the large-scale BESSs. This paper proposes a new cloud-based battery condition monitoring and fault diagnosis platform for the large-scale Li-ion BESSs. The proposed cyber-physical platform incorporates the Internet of Things embedded in the battery modules and the cloud battery management platform. Multithreads of a condition monitoring algorithm and an outlier mining-based battery fault diagnosis algorithm are built in the cloud battery management platform (CBMP). The proposed cloud-based condition monitoring and fault diagnosis platform is validated by using a cyber-physical testbed and a computational cost analysis for the CBMP. Therefore, the proposed platform will support the on-board health monitoring and provide an intelligent and cost-effective maintenance of the large-scale Li-ion BESSs.
\end{abstract}

Keywords: battery management system (BMS); cloud computing; condition monitoring; fault diagnosis; Internet of Things (IoT); large-scale lithium-ion battery energy storage systems; lithium-ion battery

\section{Introduction}

Lithium-ion (Li-ion) batteries are excellent power source and energy storage devices used in various electrical and electronic systems due to high power and energy density, low maintenance requirement, low self-discharge, and no memory effect [1]. Therefore, Li-ion batteries have recently gained increasing interest in the large-scale (MW-scale) battery energy storage systems (BESSs) which is now a critical unit for the management of electric power grids [2,3], especially with high penetration of renewable energy generations [4-6] and electric vehicles (EVs) $[7,8]$. However, there are critical concerns regarding the safety, reliability, and performance degradation of the Li-ion batteries [9] and the overall system cost is still high. A properly designed battery management systems (BMSs) are required not only for battery health monitoring and control to ensure their safety, reliability, optimal performance, but also for cost-effectiveness [10]. Especially, these challenges will be more significant as the number of battery cells increases, resulting in impeding the proliferation of large-scale Li-ion BESSs.

BMS typically includes a main BMS and module management systems for a battery bank [11,12]. A battery module mainly consists of multiple battery cells connected in series (e.g., 12-cell [13] and 96-cell with eight 12-cell monitoring integrated circuits (ICs) [14]). Figure 1 shows a grid-connected large-scale Li-ion BESS where multiple battery banks are connected to the power grid through 
converters and an energy management system (EMS) manages the overall BESS. However, several design deficiencies in current BMSs have hindered the integration of large-scale Li-ion battery systems. These deficiencies include: (1) adopting sophisticated sensing [15] and wired communication (e.g., controller area network (CAN) bus, [16], as shown in Figure 1), resulting in critical wire-harness issues such as physical connection failure, communication error under electromagnetic interference (EMI), increased cost, weight, complexity of battery pack design, and difficulty in automated manufacturing [17]; (2) lacking a real-time fault diagnosis and prognosis algorithm; and (3) lacking cost-effective embedded systems to implement the comprehensive battery algorithms (e.g., condition monitoring [18], fault diagnosis [19] and prognosis [20]) and optimal control algorithms [2], which might require high computational resources when designing the BMS systems.

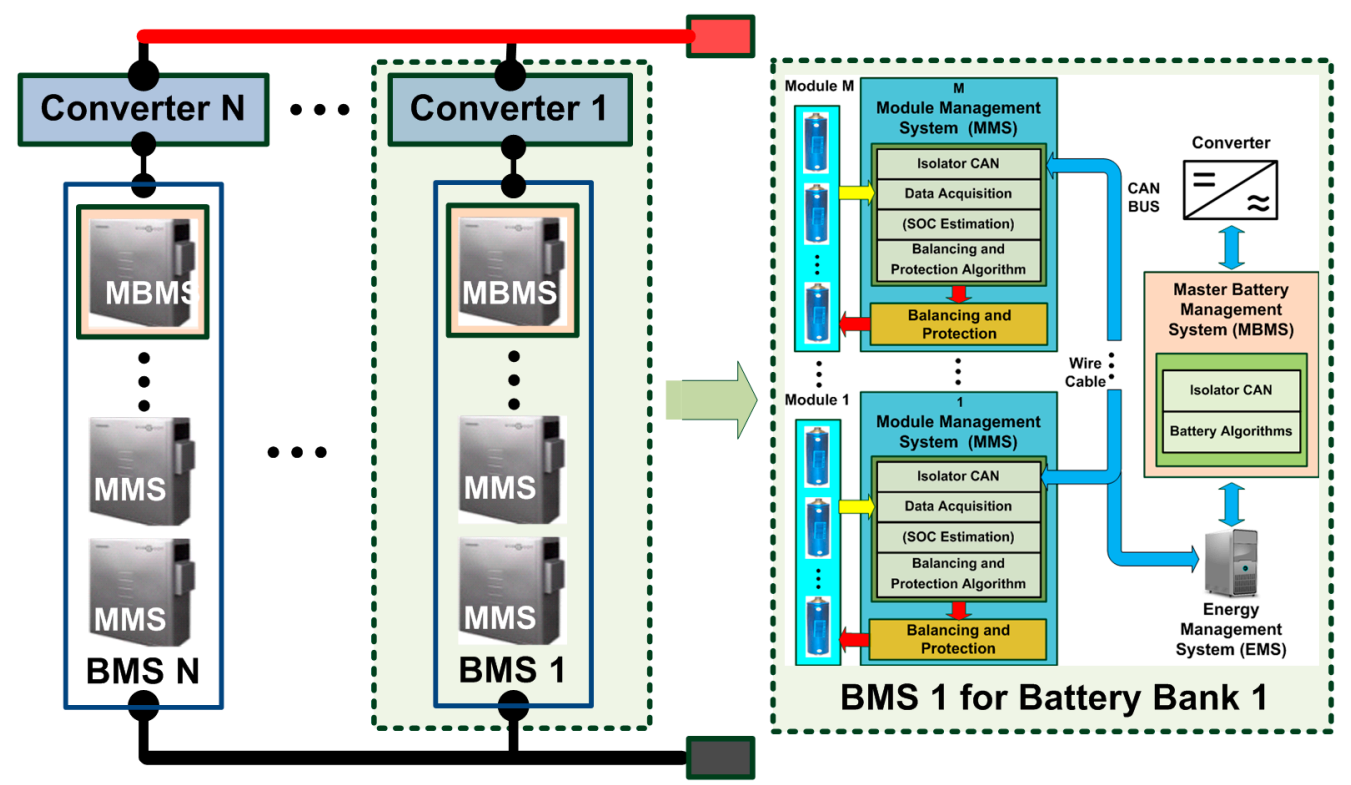

Figure 1. The conventional battery management systems (BMSs) where each BMS consists of module management systems (MMSs) and a master BMS for a large-scale Li-ion battery energy storage system (BESS).

BMS can be revolutionized as a result of further investigation of the Internet of Things (IoT) technologies [21] and cloud computing resources [22] to identify battery cell's health condition and to enhance scalability, cost-effectiveness, adaptability, safety, reliability, and flexibility of the large-scale Li-ion BESSs. Recently, a cloud connected battery management system for small EVs [23] and cloud battery data analytics services to accelerate battery product development [24] have been developed. However, the actual investigation of IoT and cloud computing to design a cyber-physical BMS platform for the large-scale Li-ion BESSs has been less studied.

A key mission of a BMS is to monitor battery health in online by a set of algorithms: condition monitoring, fault diagnosis, and fault prognosis to improve operational performance, safety, reliability, and lifespan of batteries. An excellent summary of the battery health monitoring algorithms may be found in [25]. Condition monitoring for batteries is to track changes their critical parameters and operational states (e.g., state of charge (SOC) and state of health (SOH) [26]. Unfortunately, they need to be commonly estimated using the measurable data of the battery cells (e.g., voltage, current, and surface temperature) since the values of those parameters and states are not directly measured by sensors in BMSs. In general, the model-based condition monitoring methods are used. Variety model-based condition monitoring algorithms have been proposed to estimate battery parameters and states simultaneously. Kalman filter (KF)-family has been still widely used (e.g., a dual extended KF (DEKF) [27], a dual sigma-point KF [28]) However, they increase the accuracy at the expense of higher computational effort. One major alternative of KF-family is the more computationally efficient 
observer-based approaches (e.g., dual sliding mode observer (SMO) [29]). However, the accuracy of the SMO-based methods will degrade due to the chattering when the model uncertainties are significant.

Battery cell faults include mild faults (e.g., aged faulty cell due to aging process) and incipient faults (e.g., internally shorted cell). Specifically, early detection of an internal short circuit (i.e., the status of an internal micro short circuit) in a battery cell can prevent thermal runaway that occurs a fire and an explosion, and thus ensures battery safety [30]. Fault diagnosis for battery cell is a critical technique that detects faulty cells and identifies types of faults [31]. A variety of fault diagnosis methods have been proposed, which, are generally classified into categories: model-based methods and model-free/data-driven methods. Model-based methods utilize a battery model (e.g., electrochemical models [32,33], electrical circuit models [34], multiple models [35], and thermal models [36]) and estimate parameters and/or evaluate residuals which can be good indicators for battery faults. Model-based condition monitoring algorithm have been applied for the model-based fault diagnosis (e.g., a sliding mode observer (SMO) [37], an adaptive unscented KF [38], and a structural analysis and sequential residual generators [39]). Model-free methods extract fault symptoms from signals by using signal processing methods (e.g., wavelet transform [40] and Shannon entropy [41]) and using artificial intelligence [31] (e.g., fuzzy logic and artificial neural network).

This paper proposes a novel cloud-based battery condition monitoring and fault diagnosis platform, which incorporates IoT-enabled wireless battery module management systems (WMMS) and the proposed cloud battery management platform (CBMP) to support on-board battery health monitoring and to provide intelligent and cost-effective maintenance of the large-scale BESSs. The hybrid filter (HF)-based condition monitoring algorithm of cells and the proposed outlier detection-based fault diagnosis algorithm are implemented in the CBMP. The proposed cloud-based condition monitoring and fault diagnosis platform is validated by: (1) a small-scale cloud-based BMS simulator where a small-scale CBMP is implemented in Google Cloud and three battery module emulators (BMEs) which are designed by three Raspberry pi boards (i.e., IoT devices) are built for condition monitoring and fault diagnosis of thirty battery cells; and (2) a computational cost analysis of the use of Google Cloud for the large-scale Li-ion BESSs. The results show that health conditions of individual battery cells can be accurately estimated, and abnormal cells are effectively detected in the cloud. Furthermore, the proposed platform is a scalable, fast, and cost-effective for maintenance of the large-scale Li-ion BESSs. Compared to KF-family and SMO, the HF requires less computational cost [42] and chattering issue, respectively. Furthermore, the outlier-based fault diagnosis is simple and easy to implement compared to the conventional methods.

To the authors' best knowledge, the investigation and actual implementation of the cloud-based battery health monitoring platform for the large-scale BESSs has not been studied yet, which supports on-board battery health monitoring and provides tools that will enable a new level of intelligent and cost-effective maintenance in cyber-physical environments replacing an expensive supervisory control and data acquisition system toward the next generation BMS. Furthermore, the proposed cloud platform can be easily applied to other types of energy storage systems (e.g., supercapacitor [43]-based energy storage systems). Providing such a tractable and practical cyber-physical platform for large-scale Li BESSs is the main contribution of this paper.

\section{Cloud-Based Battery Condition Monitoring and Fault Diagnosis Platform}

The conventional BMS will be remarkably advanced by fully taking the advantages of the state-of-the-art IoT and cloud computing systems toward a next generation BMS for larger-scale BESSs. Figure 2 shows an overall design concept of the cyber-physical BMS for large-scale Li-ion battery energy storage systems, which is a set of: (1) wireless module management systems (WMMSs) incorporating IoT devices; and (2) a CBMP consisting of a cloud database, an analytics tools and battery algorithms (i.e., cloud engine), and a cloud visualization. The components of the cloud-based condition monitoring and fault diagnosis platform proposed in this paper are highlighted in Figure 2. The proposed cloud-based health monitoring platform includes IoT components 
(i.e., a data acquisition, communication, and an embedded processor) in the battery modules and cloud components (i.e., a cloud storage and parallel computing, data mining analytics tools, and battery condition monitoring and fault diagnosis algorithms). It is expected that the proposed cyber-physical platform will also open research opportunities of dispersed wireless BMS systems for EVs, large transport aircraft [44], electric ships [45], and cloud-based smart grids [46].



Figure 2. The system architecture of the proposed cyber-physical battery management system for the large-scale Li-ion battery energy storage systems and components of the proposed cloud-based battery condition monitoring and fault diagnosis platform.

\subsection{IoT Components (Wireless Module Management System)}

The data acquisition is performed by sensors that measure battery cell voltage, current, and temperature at given sampling time (e.g., $T_{s}=1 \mathrm{~s}$ ) in the WMMS. The data is temporary stored in the communication component in the IoT device which is responsible for sending the stored data to the cloud data storage in the cloud server with assured security, ideally in near real-time using transmission control protocol/internet protocol (TCP/IP) protocol via IoT Gateway/router. On the other hand, the module management system receives the comprehensive health monitoring results and optimal control commends form cloud battery management system. Typically, the IoT devices is equipped with a short-range radio such as Wi-Fi, Zigbee, and Low-power Bluetooth, which transfers data/signals to other modules or a concentrator using IoT protocols [47]. Moreover, IoT Gateway is expected to aggregate large amounts of data from diverse IoT devices, with the consequent requirement for quick data aggregation, and an increase in the necessity to index, store, and process the data more effectively. Access to the cloud will be offered through various user interfaces such as IoT interface application programming interface (API). Due to the IoT and cloud support, the WMMS can reduce wire-harness issue, simpler on-board controller, resulting in increased battery module scalability and improved manufacturing productivity.

\subsection{Cloud Components (Cloud Battery Management Platform)}

Figure 3 depicts the proposed CBMP that has three distinct cloud components: cloud storage $\left(C_{1}\right)$; cloud engine $\left(C_{2}\right)$ where a cloud software platform integrates analytics tools and battery algorithms; and a cloud visualization $\left(C_{3}\right)$. The system is designed to support battery health monitoring, control, and maintenance through condition monitoring such as SOC and critical model parameters of battery cells (e.g., capacity and impedance), early detection battery cell failures (i.e., fault diagnosis), prediction of the remaining useful life of battery cells (i.e., fault prognosis), and optimal control under smart grid environment can be added. 
$\mathrm{C}_{1}$ incorporates the data storage and management tool that is offered by cloud service providers or cloud database service (e.g., Firebase offered by Google). $C_{1}$ will enable IoT or users to: (1) upload the battery data from the IoT devices in WMMSs into the cloud storage via internet; (2) assign data types (e.g., private or public data, testing conditions, battery chemistry, etc.); (3) allocate data to their storage folders in the cloud database server; and (4) send the health conditions of the individual battery cells and optimal control commands to IoT devices in WMMSs.

$C_{2}$ employs: (1) the high-performance parallel computing that executes multithreads of the high computational battery algorithms to monitor a large number of battery cells very fast; (2) data mining to find abnormal battery cells from the outcomes of condition monitoring algorithms for fault diagnosis (e.g., outlier detection [48]); (3) machine learning for fault prognosis (e.g., support vector machine); and (4) optimization tools for optimal power management (e.g., Google optimization tools (OR-Tools)) given health conditions and demand of smart grid. Additional analytical tools offered by the cloud service providers will be easily incorporated in the cloud software platform through APIs.

$\mathrm{C}_{3}$ makes the data and analytics results accessible to users in a readily digestible format such as tables, charts, and graphs. Users can utilize visualization tools offered by cloud service providers (e.g., Google chart) or by open source libraries (e.g., D3.js).

As a result, the proposed CBMP will not only dramatically reduce overall system and maintenance costs, but also improve operational performance and safety of the large-scale BESSs.



Figure 3. The proposed cloud battery management system platform.

\section{Cloud-Based Battery Condition Monitoring and Fault Diagnosis Algorithms}

Battery algorithms are a set of comprehensive algorithms for BMS, which includes condition monitoring, fault diagnosis, fault prognosis, optimal control, etc. A hybrid filter (HF)-based condition monitoring incorporating a real-time battery model developed by authors [42] and the proposed outlier mining-based fault diagnosis algorithm are applied in the CBMP. In addition, various cloud analytics tools can be easily included in the cloud platform for fault prognosis and optimization tools for optimal control under smart grid environment.

\subsection{HF-Based Condition Monitoring}

Figure 4 shows the real-time battery model. VOC (i.e., instantaneous open-circuit voltage (OCV) comprises two parts. The first part, denoted by $V_{o c}(S O C)$, represents an equilibrium OCV, which is used to bridge the SOC to the cell average OCV. The second part $V_{h}$ is the hysteresis voltage capturing the hysteresis effect of the OCV. The IV characteristics and the transient response of the battery cell are modeled by the RC circuit: Specifically, the series resistance $R_{s}$ identifies the charge/discharge energy losses of the cell; the charge transfer resistance $R_{c}$ and the double layer capacitance $C_{d}$ represent the 
short-term diffusion voltage $V_{d}$ of the cell; and $V_{\text {cell }}$ is the terminal voltage of the cell. A discrete-time state-space version of the electrical circuit with hysteresis model is described as follows [1]:

$$
\begin{array}{r}
x(k+1)=\left[\begin{array}{lll}
1 & 0 & 0 \\
0 & \alpha & 0 \\
0 & 0 & H
\end{array}\right] x(k)+\left[\begin{array}{cc}
\frac{-\eta T_{s}}{C_{\text {tot }}} & 0 \\
\beta & 0 \\
0 & (H-1)
\end{array}\right]\left[\begin{array}{c}
i_{B}(k) \\
\operatorname{sign}\left(i_{B}(k)\right)
\end{array}\right] \\
y(k)=V_{o c}(S O C(k))-V_{d}(k)-R_{s} i_{B}(k)+V_{h \max } v_{h}(k) \\
V_{o c}(S O C)=-a_{0} \exp \left(-a_{1} S O C\right)+a_{2}+a_{3} S O C-a_{4} S O C^{2}+a_{5} S O C^{3}
\end{array}
$$

where $y(k)=V_{\text {cell }}(k), x(k+1)=\left[\operatorname{SOC}(k+1), V_{d}(k+1), v_{h}(k+1)\right]^{T}$ is the state, $k$ is the time index, $V_{\text {cell }}(k)$ is the measured cell terminal voltage, $\eta$ is the Coulomb efficiency (assuming $\eta=1$ ); $C_{\text {tot }}$ denotes the total capacity of the battery cell [49], $T_{S}$ is the sampling period; $i_{B}(k)$ is the instantaneous cell current $\left(i_{B}\right.$ is negative if the cell is operated in the charge mode); $V_{h \max }$ represents the maximum hysteresis voltage; $\alpha=\exp \left(-T_{s} / \tau\right)$ with $\tau=R_{c} \cdot C_{d} ; \beta=R_{c}(1-\alpha) ; \operatorname{sign}(\cdot)$ is the sign function; and $H\left(i_{B}\right)=\exp \left(-\rho\left|i_{B}\right| T_{s}\right)$, where $\rho$ is the hysteresis parameter characterizing the convergence rate; and $a_{j}(j=0, \cdots, 5)$ are the coefficients used to parameterize the $V_{o c}(S O C)$ function. To simulate a micro shorted cell, a short circuit resistor $R_{\text {isc }}$ [34] will be included, as shown in Figure 4.

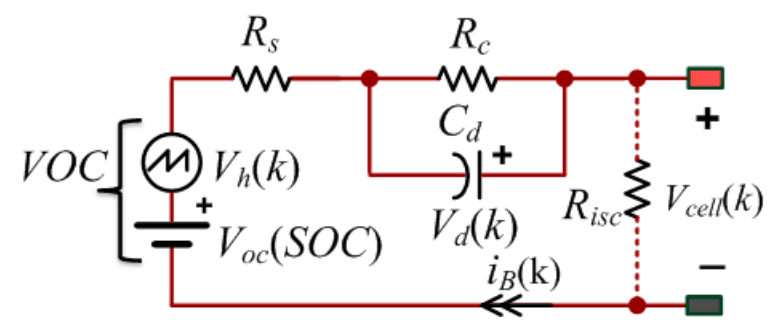

Figure 4. Battery A real-time battery model with internal short circuit resistor $R_{\text {isc }}$.

A block diagram of the HF is shown Figure 5. The essence of the HF method is to combine an extended Kalman filter (EKF) for real-time model parameter identification and a smooth variable structure filter (SVSF) for real-time SOC estimation. When new measurements are available, two filters run concurrently at each time interval: the state SVSF estimates states using the current estimated model parameters from the weighted EKF and the weighted EKF estimates the model parameters using the current estimated states from the state SVSF. Based on the prior information, the algorithm is initialized by choosing the best guesses of the battery parameters, state $x_{0}$ and tuning parameters of EKF (i.e., $P_{0}, Q, R$ ), and SVSF (i.e., $\gamma$ and $\Psi$ ).

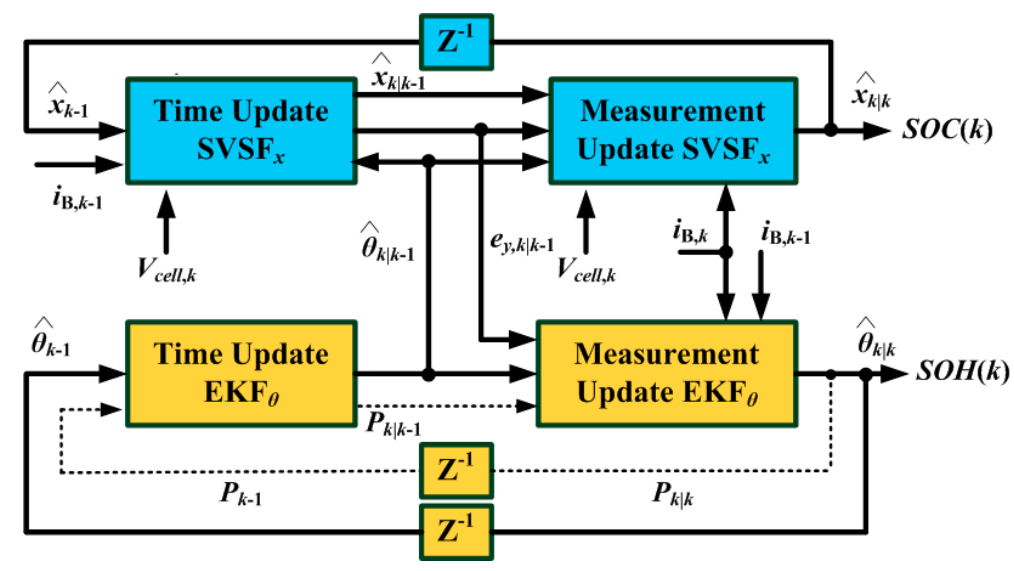

Figure 5. Block diagram of the hybrid filter. 
The parameter filter uses a state equation of cell parameters and an output cell voltage of optimal parameter dynamics.

$$
\begin{gathered}
\theta_{k+1}=\theta_{k}+r_{k} \\
y_{k}=h\left(x_{k}, i_{B, k}, \theta_{k}\right)+e_{k}
\end{gathered}
$$

where parameter vector $\theta=\left[\alpha, \beta, 1 / C_{\text {tot }}, R_{s}, \rho, V_{h \max }\right]$ and the corresponding state error covariance matrix $P ; r_{k}$ is the process noise assumed to be Gaussian white noise with zero mean and covariance of $Q$; and $e_{k}$ represents measurement noise and estimation error with zero-mean Gaussian white noise assumption and its covariance of $R$. The weighted EKF first executes time update by computing prior parameter vector $\hat{\theta}_{k \mid k-1}$ and their error covariance $P_{k \mid k-1}$.

$$
\begin{gathered}
\hat{\theta}_{k \mid k-1}=\hat{\theta}_{k-1} \\
P_{k \mid k-1}=P_{k-1}+Q
\end{gathered}
$$

Then, the estimated measurements are computed.

$$
\begin{gathered}
\left.K_{k}^{\theta}=P_{k \mid k-1}\left(C_{k}^{\theta}\right)^{T}\left[C_{k}^{\theta} P_{k \mid k-1}\left(C_{k}^{\theta}\right)^{T}+R\right)\right]^{-1} \\
\hat{\theta}_{k \mid k}=\hat{\theta}_{k \mid k-1}+K_{k}^{\theta} e_{y, k \mid k-1} \\
P_{k \mid k}=\left(I_{6}-L_{k} C_{k}^{\theta}\right) P_{k \mid k-1}
\end{gathered}
$$

where $K_{k}^{\theta}$ is Kalman filter gain at time $k$. Since $\hat{x}_{k}$ is function of the parameter vector $\hat{\theta}_{k}$, the computation of $C_{k}^{\theta}$ involves a total derivative.

The SVSF is designed based on the state space model (1) and (2) to perform the state estimation and SVSF gain equation is modified for battery cells. The dynamics of the proposed SVSF are given by:

$$
\hat{x}_{k+1 \mid k}=f\left(\hat{x}_{k}, i_{B, k}, \hat{\theta}_{k}\right)
$$

where $\hat{x}_{k+1 \mid k}$ is the predicted (or priori) state estimate, $\hat{x}_{k \mid k}$ is the current state estimate, $f$ is a vector field. The state SVSF follows the similar manner as the weighted EKF. The state SVSF first executes time update by computing prior state vector $\hat{x}_{k \mid k-1}$ and a measurement $\hat{y}_{k \mid k-1}$ is written as follows:

$$
\begin{gathered}
\hat{x}_{k \mid k-1}=f\left(\hat{x}_{k-1}, i_{B, k-1}, \hat{\theta}_{k \mid k-1}\right) \\
\hat{y}_{k \mid k-1}=h\left(\hat{x}_{k \mid k-1}, i_{B}(k), \hat{\theta}_{k \mid k-1}\right) \cong C_{k}^{x} \hat{x}_{k \mid k-1} \\
e_{y, k \mid k-1}=y_{k}-h\left(\hat{x}_{k \mid k-1}, i_{B, k}, \hat{\theta}_{k \mid k-1}\right)
\end{gathered}
$$

where $C_{k}^{x}$ is the linearized measurement matrix and written as follows:

$$
C_{k}^{x}=\left[\frac{\partial V_{O C}\left(S O C_{k \mid k-1}\right)}{\partial S O C_{k \mid k-1}}, \quad-1, \quad-1\right]
$$

To insure the numerical stability, the SVSF gain, $K_{k}^{x}$, will be also calculated as:

$$
K_{k}^{x}=\frac{\left(C_{k}^{x}\right)^{T}}{C_{k}^{x} C_{k}^{x T}+\omega I_{3}}\left(\left|e_{y, k \mid k-1}\right|+\gamma\left|e_{y, k-1}\right|\right) \circ \operatorname{sat}\left(e_{y, k \mid k-1}, \Psi\right)
$$

where $e_{y, k-1}$ represents a posteriori measurement error in the previous step; $\Psi$ denotes the smoothing boundary layer width; $\gamma(0<\gamma<1)$ is the SVSF convergence rate; $\circ$ is the Schur product; and $I_{3}$ is the 
$3 \times 3$ identity matrix; and $\omega$ is the damping parameter (e.g., $10^{-12}$ ). The SVSF gain is used to correct the state estimate $\hat{x}_{k \mid k-1}$ as follows:

$$
\left\{\begin{array}{l}
\hat{x}_{k \mid k}=\hat{x}_{k \mid k-1}+K_{k} \\
e_{y, k}=y_{k}-h\left(\hat{x}_{k \mid k}, i_{B, k}, \hat{\theta}_{k \mid k-1}\right)
\end{array}\right.
$$

where $\hat{x}_{k \mid k}$ is the corrected (or posteriori) state estimate in the current time step $k$.

\subsection{Outlier Mining-Based Fault Diagnosis}

Fault diagnosis mainly detects abnormal cells (i.e., shorted cells or anomaly aged cells) that will be faulted very soon, which are more practically useful in the battery systems. Any detectable abnormalities in the measurements (e.g., cell voltage and current) and condition monitoring results (e.g., states SOC, $V_{c}$, and $V_{h}$, and parameters $C_{\text {tot }}, R_{s}$, and $R_{c}$ ) will be good indicators of fault diagnosis algorithms. Figure 6 shows a clustering analysis of healthy cells, shorted cells [34], and anomaly aged cells (i.e., almost dead cells) $[19,20]$ using estimated parameters $C_{t o t}$ and $R_{t o t}\left(=R_{s}+R_{c}\right)$. Since the shorted cells and aged cells have high abnormalities of $C_{t o t}$ and $R_{t o t}$ compared to those of normal cells, two parameters are considered as good outlier values in the proposed method.

Outlier detection is a method of data mining and a process of finding data objects with behaviors that are very different from expectation [48]. A distance-based outlier detection approach with Z-score standardized preprocessing method [50] is proposed for battery fault diagnosis using the estimated parameters $C_{t o t}$ and $R_{t o t}$ of the individual battery cells from the threads of HF-based condition monitoring algorithms in the CBMP. The Z-score standardized method is computed by:

$$
Z_{1, n}=\frac{C_{t o t, n}-\operatorname{avg}\left(C_{t o t}\right)}{s t d\left(C_{t o t}\right)}, Z_{2, n}=\frac{R_{t o t, n}-\operatorname{avg}\left(R_{t o t}\right)}{s t d\left(R_{t o t}\right)}
$$

where $Z_{1}$ and $Z_{2}$ are the preprocessed parameters of $C_{\text {tot }}$ and $R_{t o t}$, respectively; $n$ is battery cell number $(n=1, \ldots, N)$ and $N$ denotes the number of battery cells; $\operatorname{avg}($.$) and \operatorname{std}($.$) represents the mean of the$ parameters of cells the standard deviation of the parameter, respectively.

The distance-based outlier detection method utilizes Euclidean distance between two points, which develops a flexible distance function and extracts outliers effectively. The outlier values $O\left(Z_{1}\right)$ for $C_{\text {tot }}$ and $O\left(Z_{2}\right)$ for $R_{t o t}$ are defined as the sum of Euclidean distances among a specific cell $n$ and others $(i=1, \ldots, N)$.

$$
\left\{\begin{array}{l}
O\left(Z_{1, n}\right)=\operatorname{sum}\left(\left|Z_{1, n}-Z_{1, i}\right|\right), i=1, \ldots, N \\
O\left(Z_{2, n}\right)=\operatorname{sum}\left(\left|Z_{2, n}-Z_{2, i}\right|\right), i=1, \ldots, N
\end{array}\right.
$$

Large outlier values $O\left(Z_{1}\right)$ and /or $O\left(Z_{2}\right)$ mean the cell tends to be abnormal compared to other cells. When both outlier values $O\left(Z_{1}\right)$ and $O\left(Z_{2}\right)$ of a cell are large, it will be classified as an anomaly aged cell as shown in Figure 5. On the other hand, a shorted cell will only have a large $O\left(Z_{1}\right)$. By this way, the proposed fault diagnosis algorithm classifies types of faulted battery cells. 




Figure 6. Clustering analysis of healthy (normal) cells, shorted (abnormal) cells, aged (abnormal) cells.

\section{Results}

Simulation on a cyber-physical testbed validates the proposed cloud-based condition monitoring and fault diagnosis platform. The proposed cloud platform is built in Google Cloud. In Section 4.1, a small-scale cloud-based BMS simulator for thirty battery cells is developed. The results verify the effectiveness of the cloud-based condition monitoring and fault diagnosis platform. Section 4.2 makes a computational cost analysis using date from a thousand cells to demonstrate that the proposed CBMP is scalable, fast, and cost-effect so that it is applicable to large-scale BESSs.

Across this section, the real-time battery model with/without an internal short circuit resistor in Section 2.1 (see Table 1 for completeness) is used to generate cell data; the HF-based condition monitoring and the outlier detection-based fault diagnosis algorithm are implemented in the CBMP and executed. In the HF algorithm, the tuning parameters $\gamma$ and $\Psi$ of SVSF$_{x}$ are set to be 0.1 and 0.2 ; while, the initial state covariance $P_{0}$, process noise covariance matrix $Q$, and measurement noise covariance matrix $R$, used in the $\mathrm{EKF}_{\theta}$ design are defined as diag $\left[10^{-6}, 10^{-9}, 10^{-13}, 10^{-5}, 10^{-8}, 10^{-5}\right]$, $\operatorname{diag}\left[10^{-7}, 10^{-10}, 5 \times 10^{-15}, 10^{-5}, 10^{-8}, 10^{-6}\right]$ and $0.4^{2}$, respectively. The tuning parameters are selected by trial-and-error in an effort to minimize the estimation error. We use the SOC from the Coulomb counting as the reference (true) SOC. All methods use the same current profile as shown in Figure 7 , which is corrupted by zero-mean Gaussian random noises with variances $\sigma^{2}=(0.01)^{2}$.

Table 1. Battery Model Parameters.

\begin{tabular}{cccc}
\hline $\boldsymbol{R}_{\boldsymbol{i s c}} \mathbf{( o h m )}$ & $\mathbf{3 0}$ (Shorted Cell) & $\boldsymbol{R}_{\boldsymbol{i s c}}(\mathbf{o h m})$ & Infinite (Normal and Aged Cells) \\
\hline$\rho$ & $2.47 \times 10^{-3}$ & $V_{h \max }(\mathrm{V})$ & 0.03 \\
$T_{s}$ & $1 \mathrm{~s}$ & $a_{0}$ & 0.852 \\
$a_{1}$ & 63.867 & $a_{2}$ & 3.692 \\
$a_{3}$ & 0.559 & $a_{4}$ & 0.51 \\
$a_{5}$ & 0.508 & & \\
\hline
\end{tabular}




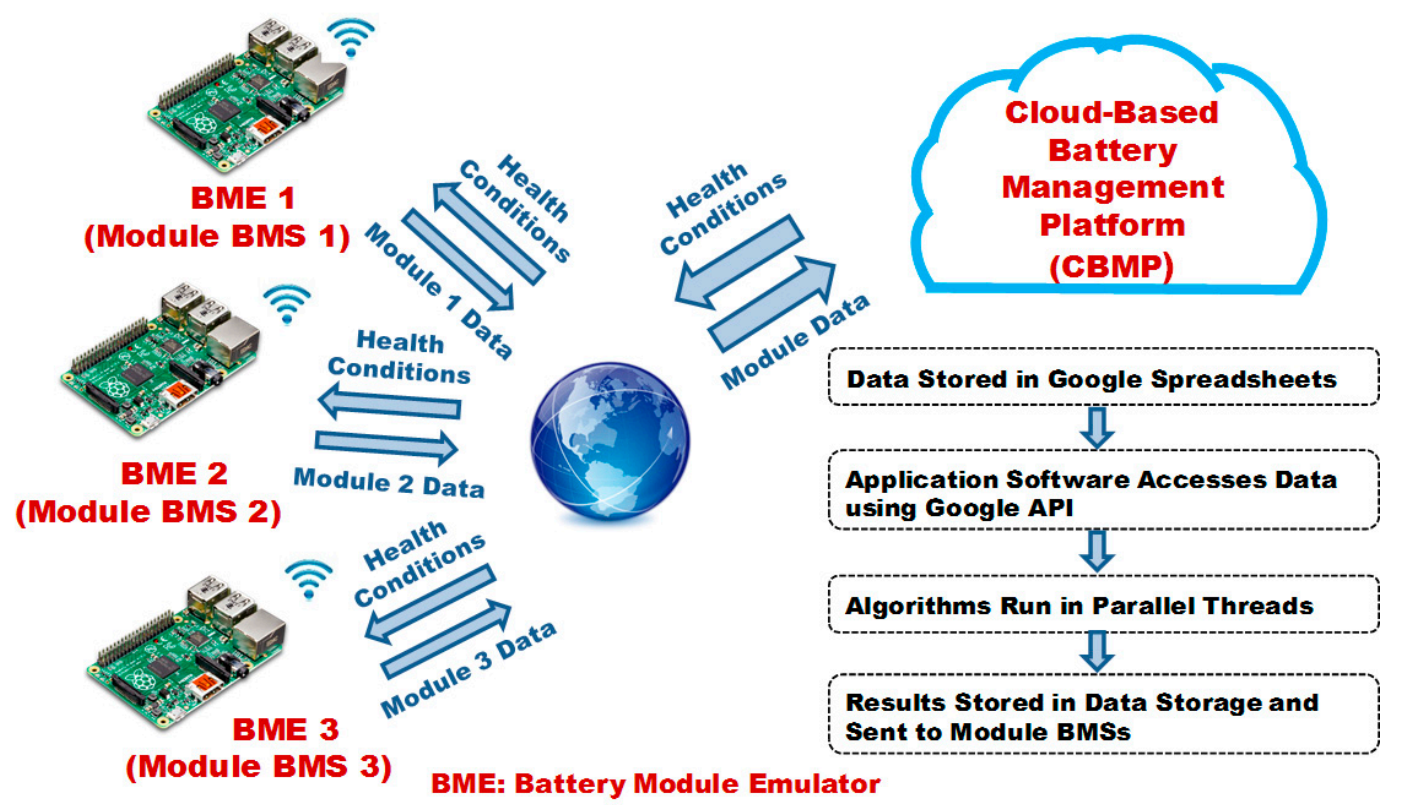

Figure 7. A Small-scale cloud-based BMS simulator.

\subsection{Validation of a Small-Scale Cloud-Based BMS}

Figure 7 illustrates the developed small-scale cloud-based BMS simulator which includes a CBMP and three battery module emulators (BME)s. The BME is designed with Raspberry pi board (i.e., an IoT device) and stores module data (i.e., battery cell voltage and current data generated by ten battery cell simulation models in Figure 3). The BMEs send the module data to Google spreadsheet (i.e., a cloud database in Figure 1) via internet, where Google sheet API embedded in the BME enables the data to be stored/synchronized in Google spreadsheet. Google App engine (i.e., an analytics tool in Figure 1) executes multithreads of the HF-based condition monitoring algorithms and the fault diagnosis algorithm written in Java for individual cells using parallel computing in the Google Cloud. The health monitoring results are stored in the Google spreadsheet, and then the health conditions are sent to module BMSs via internet and user can observe the results in the Google spreadsheet and visualization tools.

The estimation results of total capacity $C_{t}$, total resistance $R_{t}$, and SOC values of Cell 1 in BME 1 , Cell 11 in BME 2, and Cell 21 in BME 3 are validated. The results are shown in Figure 8. Figure 8b-d depict the comparison of true capacities, resistances, and SOCs of the battery cell models with the estimated values from the HF-based condition monitoring algorithm in Google cloud. The results show that the HP method converges to the true values and accurately estimate capacities, resistances, and SOCs individual battery cells except for the shorted cell. Since the battery model used in the HP-based condition monitoring does not consider the $R_{i s c}$, the estimation errors of the shorted cell are larger than others. However, this is beneficial to detect the shorted cell because the larger estimation error adds more abnormality to the outlier detector of the proposed outlier mining-based fault diagnosis algorithm. Figure $9 \mathrm{a}, \mathrm{b}$ illustrate the outlier values of the total capacities and resistance of the batteries computed by the proposed outlier mining-based fault diagnosis algorithm using the estimated $C_{\text {tot }}$ and $R_{\text {tot }}$ values. The results show that Cell 20 (aged cell) has significantly large outlier values $O\left(Z_{1}\right)$ and $O\left(Z_{2}\right)$, while Cell 10 (shorted cell) only has significantly large $O\left(Z_{1}\right)$. Therefore, the proposed fault diagnosis algorithm can provide online fault detection and classification of the individual batteries. 


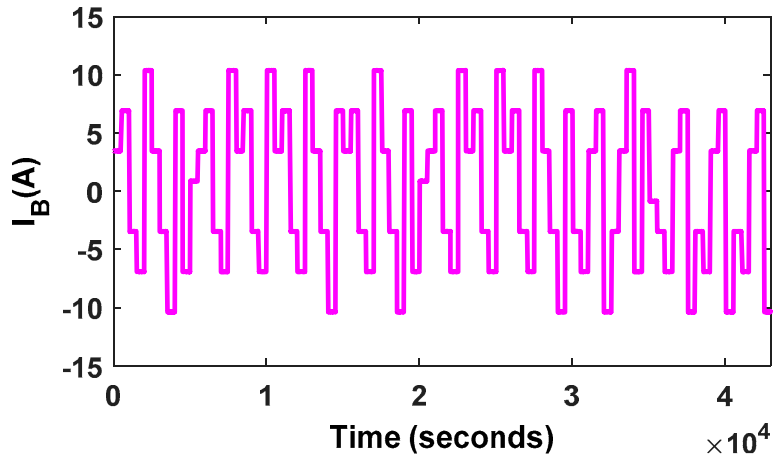

(a)

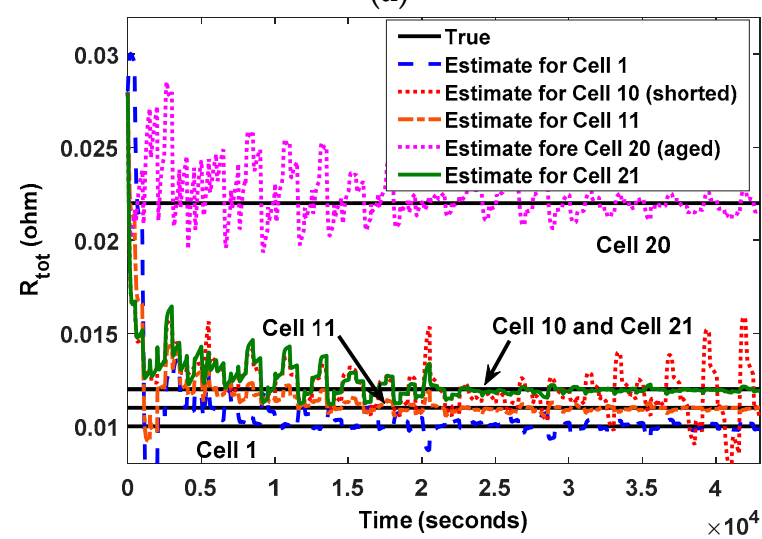

(c)

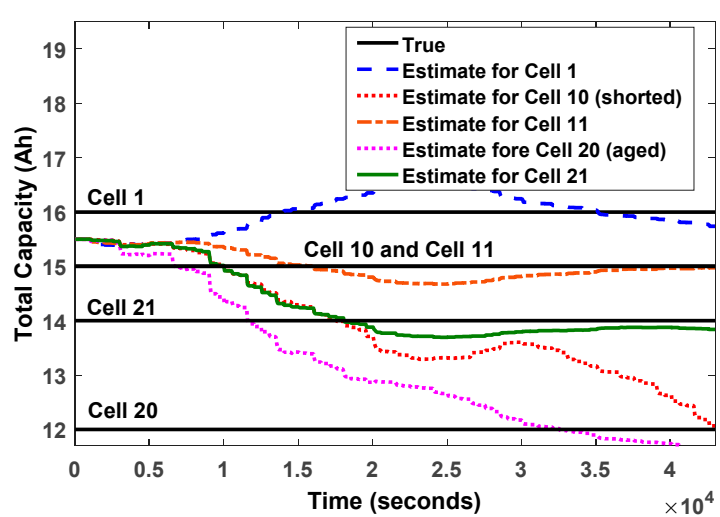

(b)

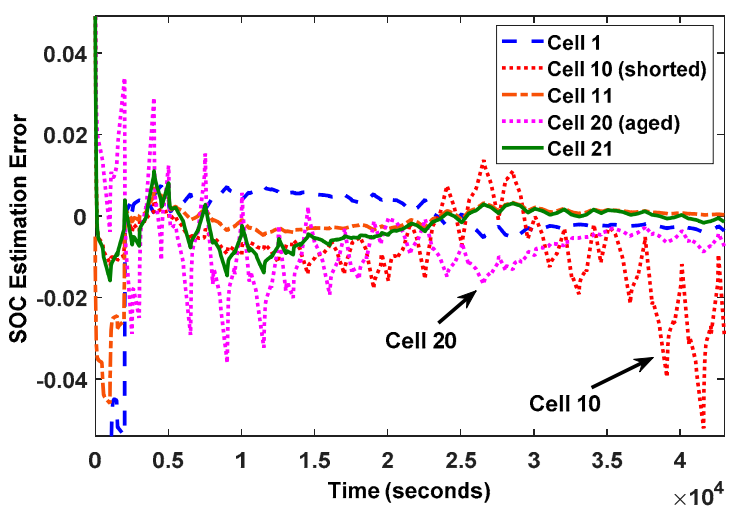

(d)

Figure 8. Comparison of true and estimated values of Cell 1, Cell 10 (shorted), Cell 11, Cell 20 (aged), and Cell 21 from the condition monitoring in Google cloud: (a) input current profile; (b) $C_{t o t}$; (c) $R_{t o t}$; and (d) state of charge (SOC) estimation error.

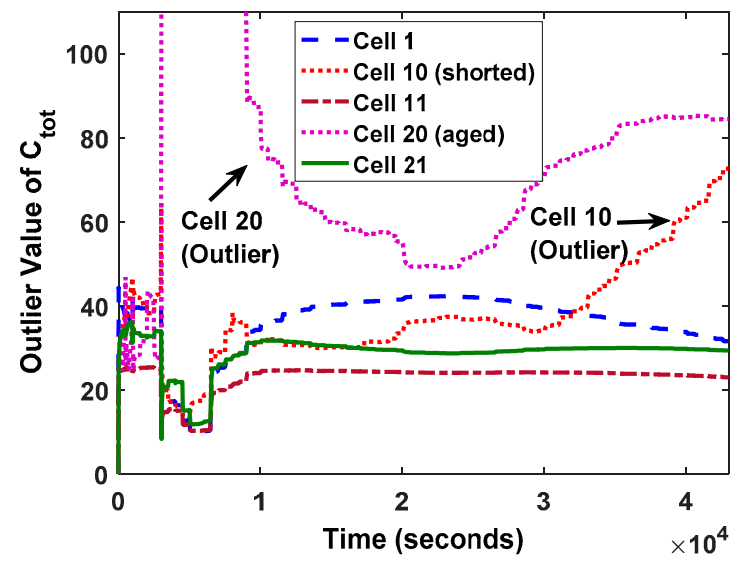

(a)

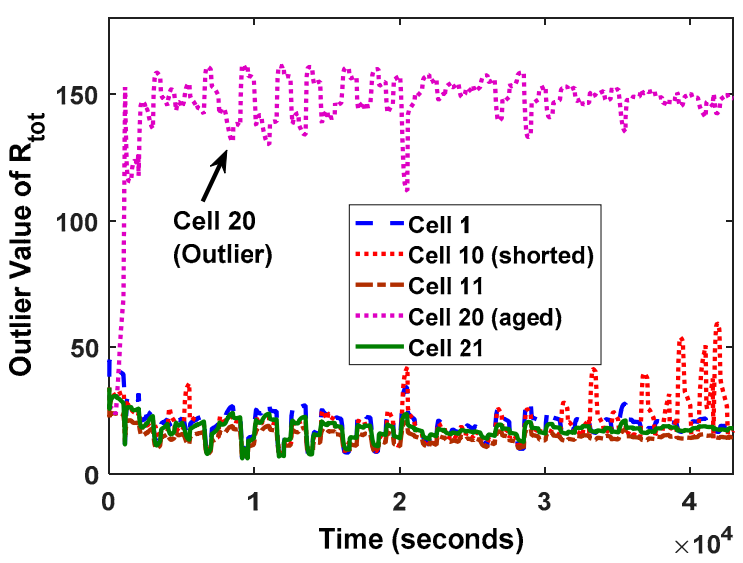

(b)

Figure 9. Outlier values of the total capacities and resistances of Cell 1, Cell 10 (internal short), Cell 11, Cell 20 (aged), and Cell 21 from the proposed outlier detection-based fault diagnosis algorithm in Google cloud: (a) $O\left(Z_{1}\right)$ and (b) $O\left(Z_{2}\right)$.

Therefore, the proposed battery condition monitoring and fault diagnosis platform enables reliable health monitoring for individual battery cells with scalable parallel computing analysis for larger-scale Li-ion battery systems, which might be impossible in the embedded BMS systems. In addition, various cloud analysis tools can be easily included in the cloud platform such as data mining tools and deep learning for the comprehensive early fault diagnosis (e.g., sensor fault, communication fault, and loose 
connection (causing arc fault)), machine learning tools for fault prognosis and optimization tools for optimal control.

\subsection{Computational Cost Analysis}

Next, we verify that the proposed CBMP works effectively in the large-scale Li-ion BESSs through computational cost analysis. Data (i.e., voltage and current) from the number of cells $(1,10,100$, and 1000) for 43,000 $\mathrm{s}$ is stored in the cloud database, where and then the battery health monitoring algorithms are executed with Intel processors (single with 3.75 GB RAM, dual with 7.5 GB RAM, and quad with 15 GB RAM) in Google Cloud. Table 2 illustrates the comparison of computational time and estimated cost for Google Cloud' three-year usage [51] of the battery health monitoring algorithms in the CBMP.

The results clearly show that the cloud platform can not only easily scale up to monitor the large number of cells by automatically developing multithreads of the health monitoring algorithm, but also compute faster by increasing the number of processor cores with additional cost. This will ultimately ensure intelligent maintenance with low cost compared to an expensive STACOM systems for the large-scale BESSs. The optimal usage of the cloud resources will depend on number of battery cells and required computation of battery algorithms and visualization, and additional software added in the CBMP, which will be further studied in the future. Therefore, the proposed CBMP can provide scalable, fast, and cost-effective health monitoring for the larger-scale Li-ion battery systems.

Table 2. Computational time in seconds and estimated cost for three-year usage of the battery health monitoring algorithm in the cloud-based battery management platform (CBMP).

\begin{tabular}{ccccc}
\hline \multirow{2}{*}{ Number of Cells } & \multirow{2}{*}{ Number of Threads } & \multicolumn{3}{c}{ Number of Cores } \\
\cline { 3 - 5 } & & Single & Dual & Quad \\
\hline 1 & 1 & $10.29 \mathrm{~s}$ & $7.10 \mathrm{~s}$ & $4.5 \mathrm{~s}$ \\
10 & 100 & $11.23 \mathrm{~s}$ & $6.35 \mathrm{~s}$ & $3.79 \mathrm{~s}$ \\
100 & 1000 & $14.56 \mathrm{~s}$ & $7.39 \mathrm{~s}$ & $4.68 \mathrm{~s}$ \\
1000 & $21.23 \mathrm{~s}$ & $13.42 \mathrm{~s}$ & $8.53 \mathrm{~s}$ \\
\hline Estimated cost for 3 years (\$) in committed usage & $\$ 561$ & $\$ 1123$ & $\$ 2247$ \\
\hline
\end{tabular}

\section{Conclusions}

This paper has proposed a cloud-based condition monitoring and fault diagnosis platform for large-scale Li-ion BESSs. The proposed platform is implemented and validated in a cyber-physical testbed using Google Cloud and Raspberry Pi 3 IoT boards. The results show that SOCs, resistances, and capacities of individual battery cells can be accurately estimated by the multithread of condition monitoring algorithms as well as faulty cells can be detected by the data mining algorithm in the proposed platform. Moreover, computational cost analysis has validated that the proposed platform is scalable, fast, and cost-effective health monitoring for the larger-scale Li-ion battery systems. Future works include: (1) the design of the WMMS hardware and effective wireless IoT network; (2) improving CBMP by adding more battery algorithms, optimal control methods, and visualization tools; (3) evaluating the impact of network latency of cloud-based communications on the BMS, (4) overall life time cost analysis and optimal cloud resource allocation of the proposed platform and (5) analysis of data obtained from an actual large-scale BESS. As a result, the advanced BMS will not only dramatically reduce the barriers of impeding the proliferation of large-scale Li-ion BESSs by reducing the overall system and maintenance costs, but also improve the operational performance and safety of the large-scale battery systems.

Author Contributions: Taesic Kim suggested the main idea of this manuscript and designed the algorithms. Amit Adhikaree contributed to developing the small-scale cloud-based BMS simulator. Darshan Makwana 
designed the cloud platform. Jitendra Shamjibhai Vagdoda conducted cloud test. Young Lee supervised the cloud work and reviewed the paper.

Conflicts of Interest: The authors declare no conflict of interest.

\section{References}

1. Scrosati, B.; Garche, J. Lithium batteries: Status, prospects and future. J. Power Sources 2010, 195, $2419-2430$. [CrossRef]

2. Roberts, B.P.; Sandberg, C. The role of energy storage in development of smart grids. Proc. IEEE 2011, 99, 1139-1144. [CrossRef]

3. Kenning, T. LG Chem's 1 GWh 'Large Contract' with AES Worth US \$300 Million. 2015 Energy Storage News Media. Available online: http:/ / www.energy-storage.news/news/lg-chem-signs-its-largestsupplycontract-for-stationary-storage-with-aes-e (accessed on 1 November 2017).

4. Bragard, M.; Soltau, N.; Thomas, S.; de Doncker, R.W. The balance of renewable sources and user demands in grids: Power electronics for modular battery energy storage systems. IEEE Trans. Power Electron. 2010, 25, 3049-3056. [CrossRef]

5. Neubauer, J.; Simpson, M. Deployment of Behind-The-Meter Energy Storage for Demand Charge Reduction; Technical Report NREL/TP-5400-63162; National Renewable Energy Lab. (NREL): Golden, CO, USA, 2015; pp. 1-22.

6. Wu, L.; Gao, W.; Cui, Z.; Kou, X. A novel frequency regulation strategy with the application of energy storage system for large scale wind power integration. In Proceedings of the IEEE Green Technologies Conference, New Orleans, LA, USA, 15-17 April 2015; pp. 221-226.

7. Dinger, A.; Martin, R.; Mosquet, X.; Rabi, M.; Rizoulis, D.; Russo, M.; Sticher, G. Batteries for electric cars-challenges: Opportunities and the outlook to 2020. Energy Environ. Publ. 2010, 87, 18-23.

8. Neubauer, J.; Pesaran, A. The ability of battery second use strategies to impact plug-in electric vehicle prices and serve utility energy storage applications. J. Power Sources 2011, 196, 10351-10358. [CrossRef]

9. Lu, L.; Han, X.; Li, J.; Hua, J.; Ouyang, M. A review on the key issues for lithium-ion battery management in electric vehicles. J. Power Sources 2013, 226, 272-288. [CrossRef]

10. Li, J.; Zhou, S.; Han, Y. Advances in Battery Manufacturing, Service, and Management Systems; Wiley: Hoboken, NJ, USA, 2016.

11. Kim, T.; Qiao, W.; Qu, L. A Multicell battery system design for electric and plug-in hybrid electric vehicles. In Proceedings of the 2012 IEEE International Electric Vehicle Conference, Greenville, SC, USA, 4-8 March 2012; pp. 1-7.

12. Andrea, D. Battery Management Systems for Large Lithium-Ion Battery Packs; Artech House: Norwood, MA, USA, 2010.

13. Texas Instrument. PowerLAN Gateway Battery Management Controller with Power Pump Cell Balancing; Electronic Publication: BQ78PL114 Datasheet; Texas Instrument: Dallas, TX, USA, 2009.

14. Linear Technology Corporation. Multicell Battery Stack Monitor; Electronic Publication: LTC6803-2/LTC6803-4 Datasheet; Linear Technology Corporation: Milpitas, CA, USA, 2011.

15. Kim, H.; Shin, K.G. Efficient sensing matters a lot for large-scale batteries. In Proceedings of the IEEE/ACM International Conference on Cyber-Physical Systems, Chicago, IL, USA, 12-14 April 2011; pp. 197-205.

16. Weicker, P. A Systems Approach to Lithium-Ion Battery Management; Artech House: Norwood, MA, USA, 2014.

17. Lee, M.; Lee, J.; Lee, I.L.J.; Chon, A. Wireless battery management system. In Proceedings of the International Battery, Hybrid and Fuel Cell Electric Vehicle Symposium, Barcelona, Spain, 17-20 November 2103; pp. 1-5.

18. Kim, T.; Wang, Y.; Sahinoglu, Z.; Wada, T.; Hara, S.; Qiao, W. Model-based condition monitoring for lithium-ion batteries. J. Power Sources 2015, 295, 16-27. [CrossRef]

19. Wu, C.; Zhu, C.; Ge, Y.; Zhao, Y. A diagnosis approach for typical faults of lithium-ion battery based on extended Kalman filter. Int. J. Electrochem. Sci. 2016, 11, 5289-5301. [CrossRef]

20. Si, X.; Wang, W.; Hu, C.; Zhou, D. Remaining useful life estimation-A review on the statistical data driven approaches. Eur. J. Oper. Res. 2010, 213, 1-14. [CrossRef]

21. Vermesan, O.; Friess, P. Internet of Things-From Research and Innovation to Market Development; River Publishers: Gistrup, Denmark, 2014.

22. Furtht, B.; Escalante, A. Handbook of Cloud Computing; Springer: New York, NY, USA, 2010. 
23. Tanizawa, T.; Szumiya, T.; Ikeda, K. Cloud-connected battery management system supporting e-mobility. Fujitsu Sci. Tech. J. 2015, 51, 27-35.

24. Voltaiq. Available online: https://www.voltaiq.com/ (accessed on 1 November 2017).

25. Rezvanizaniani, S.M.; Liu, Z.; Chen, Y.; Lee, J. Review and recent advances in battery health monitoring and prognostics technologies for electric vehicle (EV) safety and mobility. J. Power Sources 2014, 256, 110-124. [CrossRef]

26. Kim, T.; Qiao, W.; Qu, L. Online SOC and SOH estimation for multicell lithium-ion batteries based on an adaptive hybrid battery model and sliding-mode observer. In Proceedings of the 2013 IEEE Energy Conversion Congress and Exposition, Denver, CO, USA, 15-19 September 2013; pp. 292-298.

27. Plett, G.L. Extended Kalman filtering for battery management systems of LiPB-based HEV battery packs: Part 3. Sate and parameter estimation. J. Power Sources 2004, 134, 277-292. [CrossRef]

28. Plett, G.L. Sigma-point Kalman filtering for battery management systems of LiPB-based HEV battery packs: Part 2: Simultaneous state and parameter estimation. J. Power Sources 2006, 161, 1369-1384. [CrossRef]

29. Kim, I. A technique for estimating the state of health of lithium batteries through a dual-sliding-mode observer. IEEE Trans. Power Electron. 2010, 25, 1013-1022.

30. Wang, Q.; Ping, P.; Zhao, X.; Chu, G.; Sun, J.; Chen, C. Thermal runaway caused fire and explosion of lithium ion battery. J. Power Sources 2012, 208, 210-224. [CrossRef]

31. Wu, C.; Zhu, C.; Ge, Y.; Zhao, Y. A review on fault mechanism and diagnosis approach for Li-ion batteries. J. Nanomater. 2015, 2015. [CrossRef]

32. Rahman, M.A.; Anwar, S.; Izadian, A. Electrochemical model based fault diagnosis of lithium ion battery. Adv. Automob. Eng. 2016, 5, 1-9. [CrossRef]

33. Dey, S.; Ayalew, B. A diagnostic scheme for detection, isolation and estimation of electrochemical faults in Lithium-ion cells. In Proceedings of the ASME 2015 Dynamic System and Control Conference, Columbus, OH, USA, 28-30 October 2015.

34. Ouyang, M.; Zhang, M.; Feng, X.; Lu, L.; Li, J.; He, X.; Zheng, Y. Internal short circuit detection for battery pack using equivalent parameter and consistency method. J. Power Sources 2015, 294, 272-283. [CrossRef]

35. Singh, A.; Izadian, A.; Anwar, S. Fault diagnosis of Li-Ion batteries using multiple-model adaptive estimation. In Proceedings of the IEEE 39th Industrial Electronics Society Annual Conference, Vienna, Austria, 10-13 November 2013.

36. Dey, S.; Biron, Z.A.; Tatipamula, S.; Das, N.; Mohon, S.; Ayalew, B.; Pisu, P. Model-based real-time thermal fault diagnosis of Lithium-ion batteries. Control Eng. Pract. 2016, 56, 37-48. [CrossRef]

37. Marcicki, J.; Simona, O.; Rizzoni, G. Nonlinear fault detection and isolation for a lithium-ion battery management system. In Proceedings of the ASME 2010 Dynamic Systems and Control Conference, Cambridge, MA, USA, 12-15 September 2010; Volume 1.

38. Zheng, C.; Ge, Y.; Chen, Z.; Huang, D.; Liu, J.; Zho, S. Diagnosis Method for Li-ion battery fault based on an adaptive unscented Kalman filter. Energies 2017, 10, 1810. [CrossRef]

39. Liu, Z.; Ahmed, Q.; Rizzoni, G.; He, H. Fault detection and isolation for lithium-ion battery system using structural analysis and sequential residual generation. In Proceedings of the ASME 7th Dynamic Systems and Control, San Antonio, TX, USA, 22-24 October 2014.

40. Kim, J.; Cho, B.H. An innovative approach for characteristic analysis and state-of-health diagnosis for a Li-ion cell based on the discrete wavelet transform. J. Power Sources 2014, 260, 115-130. [CrossRef]

41. Hong, J.; Wang, Z.; Liu, P. Voltage fault precaution and management of lithium-ion batteries based on entropy for electric vehicles. Energy Procedia 2016, 104, 44-49. [CrossRef]

42. Kim, T.; Adhikaree, A.; Kang, D.; Kim, M.; Baek, J. A new hybrid filter-based online condition monitoring for lithium-ion batteries. In Proceedings of the 2017 IEEE International Conference on Electro Information Technology, Lincoln, NE, USA, 14-17 May 2017; pp. 22-27.

43. Genc, R.; Alas, M.O.; Harputlu, E.; Repp, S.; Kremer, N.; Castellano, M.; Colak, S.G.; Ocakoglu, K.; Erdem, E. High-capacitance hybrid supercapacitor based on multi-colored fluorescent carbon-dots. Sci. Rep. 2017, 7. [CrossRef] [PubMed]

44. Wheeler, P. Technology for the more and all electric aircraft of the future. In Proceedings of the IEEE International Conference on Automatica, Curico, Chile, 19-21 October 2016; pp. 1-5. 
45. Khan, M.M.S.; Faruque, M.O. Energy storage management for MVDC power system of all electric ship under different load condition. In Proceedings of the Electric Ship Technologies Symposium, Arlington, VA, USA, 14-17 August 2017; pp. 192-199.

46. Luo, F.; Zhao, J.; Dong, Z.Y.; Chen, Y.; Xu, Y.; Zhang, X.; Wong, K.P. Cloud-based information infrastructure for next-generation power grid: Conception, architecture, and applications. IEEE Trans. Smart Grid 2015, 7, 1896-1912. [CrossRef]

47. Al-Fuqaha, A.; Guizani, M.; Mohammadi, M.; Aledhari, M.; Ayyash, M. Internet of Things: A survey on enabling technologies, protocols, and applications. IEEE Commun. Surv. Tutor. 2015, 17, 2347-2376. [CrossRef]

48. Han, J.; Kamber, M.; Pei, J. Data Mining: Concepts and Techniques, 3rd ed.; Elsevier: Amsterdam, The Netherlands, 2011.

49. Plett, G.L. Recursive approximate weighted total least squares estimation of battery cell total capacity. J. Power Sources 2011, 196, 2319-2331. [CrossRef]

50. Angiulli, F.; Basta, S.; Pizzuti, C. Distance-based detection and prediction of outliers. IEEE Trans. Knowl. Data Eng. 2006, 18, 145-160. [CrossRef]

51. Google Cloud Pricing Calculator in July 2017. Available online: https://cloud.google.com/products/ calculator/ (accessed on 15 July 2017).

(C) 2018 by the authors. Licensee MDPI, Basel, Switzerland. This article is an open access article distributed under the terms and conditions of the Creative Commons Attribution (CC BY) license (http:/ / creativecommons.org/licenses/by/4.0/). 